\title{
On Meridional Circulation in Stars
}

\author{
Suzanne Talon, Georges Michaud and Alain Vincent \\ Département de Physique, Université de Montréal and CERCA, Canada
}

\begin{abstract}
Even though the existence of meridional currents in stars has been known for quite a long time (Eddington 1925, Vogt 1925), its exact structure as well as its influence on stellar evolution is still unclear. Some authors concentrated on finding the exact shape of meridional circulation in a rotating star, while others tried to model its effect on the chemical distribution in the interior. In all studies performed so far however, meridional circulation is considered in an asymptotic regime in which the advection of entropy by the meridional currents is supposed to balance exactly the source term of the non-zero radiative flux divergence. Other terms could however be added to that asymptotic regime which could turn out to dominate the transport of chemicals. We wish to present here preliminary results of 3D numerical simulation attempted to tackle this problem.
\end{abstract}

\section{Calculation hypothesis}

We are solving the full Navier-Stokes equations of hydrodynamics in the anelastic approximation, which consists of replacing the mass conservation equation by $\vec{\nabla} \cdot(\rho \vec{v})=0$, thus filtering out sound waves. The non-dimensionnal equations to solve are then expressing conservation of mass

$$
\vec{\nabla} \cdot(\rho \vec{v})=0
$$

and momentum

$$
\frac{\partial(\rho \vec{v})}{\partial t}+\vec{\nabla} \cdot\left(\rho \vec{v} \otimes \vec{v}+\mathcal{N}_{1} P \overline{\bar{I}}-\frac{1}{\mathcal{R} e} \overline{\bar{\tau}}\right)=\frac{1}{\mathcal{F} r} \rho \vec{g}_{\mathrm{eff}}-\frac{1}{\mathcal{R} o} \rho \vec{e}_{z} \times \vec{v}
$$

and the evolution of entropy

$$
\rho T \frac{\partial s}{\partial t}+\rho T \vec{\nabla} s \cdot \vec{v}=\left(\mathcal{N}_{2} \overline{\bar{\tau}} \cdot \vec{\nabla}\right) \vec{v}+\vec{\nabla} \cdot\left(\frac{\chi}{\mathcal{R} e \mathcal{P} r} \vec{\nabla} T\right) .
$$

The full time-dependent set of equations is then solved using the finite volume method. This numerical problem is solved on a Cartesian grid; the star is simply put in a box. The solution is calculated for all points inside the star and fictitious points (outside the star) are used so that the boundary conditions are satisfied exactly at the stellar surface. The advantage of this method is that the discretized form of the equations remains quite simple. Furthermore, it allows to deal with changes in the shape of the star by simply redefining the position of the surface. 

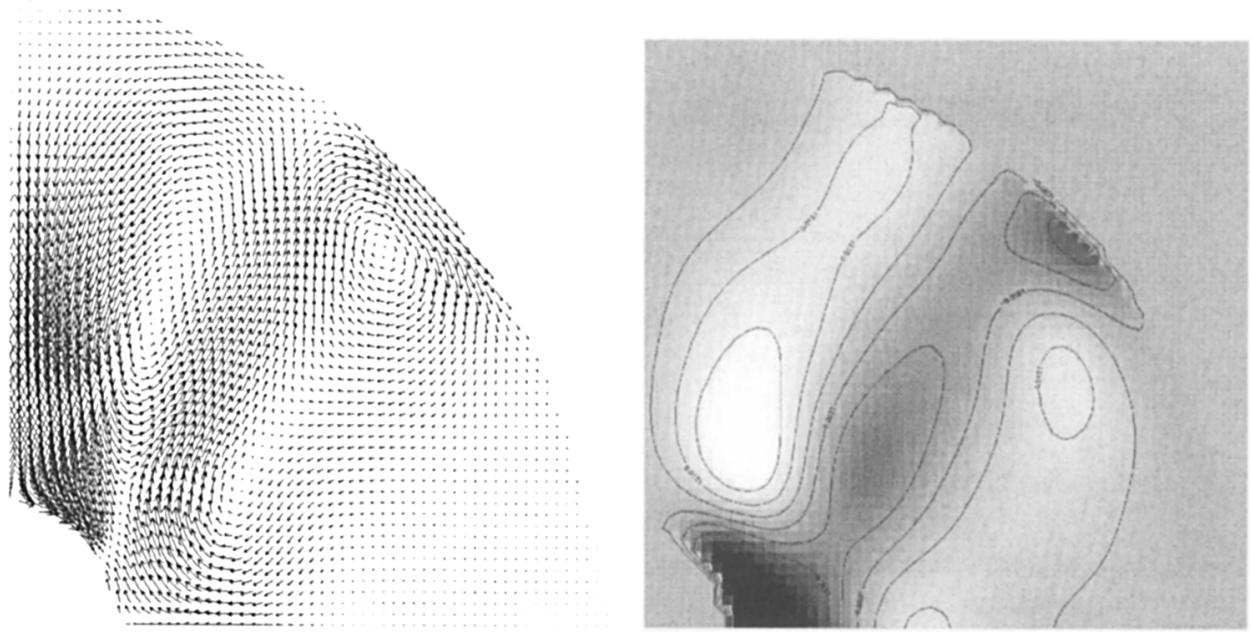

Figure 1. (left) Transitory meridionnal currents (right) Differential rotation in a meridian section

\section{Preliminary results}

Test simulations are currently performed at low spatial resolution in order to test the code. Results are shown for a $64 \times 64 \times 64$ simulation where only $1 / 8$ of the star is simulated (symmetry about the equator is assumed and the flows going out from the $x=0$ plane are injected back into the $y=0$ plane).

The values used in this simulation are $\mathcal{R} e=100, \mathcal{F} r=5, \mathcal{P} r=0.01, \mathcal{R} o$ $=1, \mathcal{A}=0.03, \mathcal{N}_{1}=1, \mathcal{N}_{2}=10^{-6}$.

Figure 1 illustrates the meridionnal currents generated within the star due to thermal imbalance some time into the simulation (no assymptotic regime has been reached yet) as well as the amount of differential rotation at this point. The Coriolis force included in the calculation breaks the initial cell of meridionnal circulation in many smaller ones. Higher resolution simulations will permit to get closer to stellar values. However, simulating a real star will require a sub-grid model, which describes the smallest scales that are not resolved directly by the code. One then hopes to gain insight into the amount of mixing which can be generated in a star due to that circulation as well as to various hydrodynamical instabilities which can be triggered by the resulting differential rotation.

\section{References}

Eddington A.S., 1925, Observatory 48, 78

Vogt H., 1925, Astron. Nachr. 223, 229 